\title{
Quench simulations for superconducting elements in the LHC accelerator
}

\author{
F. Sonnemann ${ }^{\mathrm{a}}$ \\ ${ }^{a}$ III. Phys. Inst., RWTH Aachen, Germany; LHC Div., CERN, Switzerland \\ R. Schmidt ${ }^{b}$ \\ ${ }^{\mathrm{b}}$ LHC Div., CERN, Switzerland
}

\begin{abstract}
The design of the protection system for the superconducting elements in an accelerator such as the Large Hadron Collider (LHC), now under construction at CERN, requires a detailed understanding of the thermo-hydraulic and electrodynamic processes during a quench. A numerical program (SPQR - Simulation Program for Quench Research) has been developed to evaluate temperature and voltage distributions during a quench as a function of space and time. The quench process is simulated by approximating the heat balance equation with the finite difference method in presence of variable cooling and powering conditions. The simulation predicts quench propagation along a superconducting cable, forced quenching with heaters, impact of eddy currents induced by a magnetic field change, and heat transfer through an insulation layer into helium, an adjacent conductor or other material. The simulation studies allowed a better understanding of experimental quench data and were used for determining the adequate dimensioning and protection of the highly stabilised superconducting cables for connecting magnets (busbars), optimising the quench heater strip layout for the main magnets, and studying quench back by induced eddy currents in the superconductor. After the introduction of the theoretical approach, some applications of the simulation model for the LHC dipole and corrector magnets are presented and the outcome of the studies is compared with experimental data.
\end{abstract}

Key words: AC losses, heat transfer, propagation velocity, quench, superconducting magnets 


\begin{tabular}{|c|c|c|}
\hline$A$ & cross-section & {$\left[\mathrm{m}^{2}\right]$} \\
\hline$A_{c u}$ & copper cross-section & {$\left[\mathrm{m}^{2}\right]$} \\
\hline$A_{s c}$ & niobium-titanium cross-section & {$\left[\mathrm{m}^{2}\right]$} \\
\hline$A_{h}$ & heater strip cross-section & {$\left[\mathrm{m}^{2}\right]$} \\
\hline$B$ & magnetic field & [tesla] \\
\hline$B_{\|}$ & field component parallel to the conductor block & [tesla] \\
\hline$B_{\perp}$ & field component perpendicular to the conductor block & [tesla] \\
\hline$C_{h}$ & heater power supply capacitance & {$[\mathrm{F}]$} \\
\hline$c$ & heat capacity (averaged for conductor) & {$\left[\mathrm{J} /\left(\mathrm{m}^{3} \cdot \mathrm{K}\right)\right]$} \\
\hline$c_{h}$ & heater strip heat capacity & {$\left[\mathrm{J} /\left(\mathrm{m}^{3} \cdot \mathrm{K}\right)\right]$} \\
\hline$c_{\text {iso }}$ & insulation layer heat capacity & {$\left[\mathrm{J} /\left(\mathrm{m}^{3} \cdot \mathrm{K}\right)\right]$} \\
\hline$d_{f}$ & filament diameter & {$[\mathrm{m}]$} \\
\hline$f$ & fraction of conductor exposed to helium & \\
\hline$f_{h}$ & fraction of heater energy directed into the superconductor & \\
\hline$F_{t}$ & heat transfer function for transverse cooling & {$[\mathrm{W} / \mathrm{m}]$} \\
\hline$\phi$ & angle of magnetic field and the conductor block & {$[\mathrm{rad}]$} \\
\hline$g$ & logical function that determines whether element is covered by heater & \\
\hline$h$ & height of Rutherford cable & [m] \\
\hline$h^{H e}$ & heat transfer coefficient to helium & {$\left[\mathrm{W} / \mathrm{m}^{2}\right]$} \\
\hline I & excitation current & {$[\mathrm{A}]$} \\
\hline$I_{i f}$ & interfilament coupling current & {$[\mathrm{A}]$} \\
\hline$I_{\text {is }}$ & interstrand coupling current & [A] \\
\hline$I_{h}$ & heater current & {$[\mathrm{A}]$} \\
\hline$I_{h}^{0}$ & initial heater current & {$[\mathrm{A}]$} \\
\hline$I_{0}$ & initial excitation current & [A] \\
\hline$J_{c}$ & critical current density for the conductor & {$\left[\mathrm{A} / \mathrm{m}^{2}\right]$} \\
\hline$j$ & heat flux & {$\left[\mathrm{W} / \mathrm{m}^{3}\right]$} \\
\hline$k$ & thermal conductivity & {$[\mathrm{W} /(\mathrm{m} \cdot \mathrm{K})]$} \\
\hline$k_{i s o}$ & thermal conductivity of the insulation layer & {$[\mathrm{W} /(\mathrm{m} \cdot \mathrm{K})]$} \\
\hline$k_{i s o}^{h}$ & thermal conductivity of the heater insulation layer & {$[\mathrm{W} /(\mathrm{m} \cdot \mathrm{K})]$} \\
\hline$k_{i s o}^{t}$ & thermal conductivity of the insulation between turns & {$[\mathrm{W} /(\mathrm{m} \cdot \mathrm{K})]$} \\
\hline$l_{h}^{l}$ & length (longitudinal) of heater strip & {$[\mathrm{m}]$} \\
\hline$l_{h}^{C u}$ & length of copper plated part per period of the heater strip & [m] \\
\hline$l_{h}^{h} e$ & length of non copper plated part per period of the heater strip & [m] \\
\hline$l_{\text {iso }}$ & thickness of the insulation layer & [m] \\
\hline$L_{p_{f}}$ & twist pitch length of a filament & [m] \\
\hline$L_{p_{s}}$ & twist pitch length of a strand & [m] \\
\hline$M$ & magnetisation loss & {$\left[\mathrm{W} / \mathrm{m}^{3}\right]$} \\
\hline$N_{s, l}$ & number of strands in one layer of the Rutherford cable & \\
\hline$P$ & effective wetted perimeter of the conductor & {$[\mathrm{m}]$} \\
\hline$P_{i f}$ & power loss in strands (interfilament) & {$[\mathrm{W}]$} \\
\hline$P_{i s}$ & power loss in the Rutherford cable (interstrand) & [W] \\
\hline$P 0$ & initial heater power dissipation & {$[\mathrm{W}]$} \\
\hline$\dot{\mathrm{q}}$ & heat pulse provoking the natural quench & {$[\mathrm{W} / \mathrm{m}]$} \\
\hline$R$ & resistance & {$[\Omega]$} \\
\hline$R_{a}$ & contact resistance between adjacent strands in one layer & {$[\Omega]$} \\
\hline$R_{c}$ & inter layer contact resistance & {$[\Omega]$} \\
\hline$R_{h}$ & heater strip resistance & {$[\Omega]$} \\
\hline$\rho_{c u}$ & copper resistivity & {$[\Omega \mathrm{m}]$} \\
\hline & heater resistivity (stainless steel dominated) & {$[\Omega \mathrm{m}]$} \\
\hline$R R R$ & ratio of copper resistivity at $300 \mathrm{~K}$ and $10 \mathrm{~K}$ & \\
\hline$r_{c u / s c}$ & ratio of copper to niobium-titanium content & \\
\hline & radius of the conductor & {$[\mathrm{m}]$} \\
\hline$T$ & temperature & {$[\mathrm{K}]$} \\
\hline$T_{\text {bath }}$ & helium bath temperature & {$[\mathrm{K}]$} \\
\hline$T_{\text {crit }}$ & critical temperature & {$[\mathrm{K}]$} \\
\hline$T_{c s}$ & current sharing temperature & {$[\mathrm{K}]$} \\
\hline$T_{h}$ & heater strip temperature & {$[\mathrm{K}]$} \\
\hline$T_{i s o}$ & insulation layer temperature (at boundary to helium bath) & {$[\mathrm{K}]$} \\
\hline$T_{n b}$ & temperature at which nucleate boiling starts & {$[\mathrm{K}]$} \\
\hline$T_{f b}$ & temperature at which film boiling helium starts & {$[\mathrm{K}]$} \\
\hline$t$ & time & {$[\mathrm{s}]$} \\
\hline$t_{\text {det }}$ & quench detection time & {$[\mathrm{s}]$} \\
\hline$t_{f b}$ & time interval needed to start film boiling & {$[\mathrm{s}]$} \\
\hline$\tau_{h}$ & heater current decay time constant & {$[\mathrm{s}]$} \\
\hline$\tau_{i f}$ & interfilament current build-up time constant & {$[\mathrm{s}]$} \\
\hline$\tau_{\text {is }}$ & interstrand current build-up time constant & [s] \\
\hline$U_{h}^{0}$ & initial heater voltage & [V] \\
\hline$U_{\min }^{n}$ & minimum heater voltage to provoke a quench & {$[\mathrm{V}]$} \\
\hline$V$ & volume element & {$\left[\mathrm{m}^{3}\right]$} \\
\hline$w_{h}$ & width of heater strip & {$[\mathrm{m}]$} \\
\hline$w_{t}$ & averaged turn width & [m] \\
\hline$x$ & longitudinal direction along the conductor & [m] \\
\hline$y$ & transverse direction between layers & [m] \\
\hline$z$ & transverse direction between turns & [m] \\
\hline
\end{tabular}




\section{Introduction}

Analytic models of the quench process are limited in precision since they often disregard the temperature dependence of the material parameters, assume a simple geometry and do not take the cooling by helium into account. Tests of superconducting equipment is time consuming and expensive, and the results can frequently only be understood with the help of simulation studies. As most quench experiments are performed on prototype magnets and busbars, computations are required to extrapolate to the configuration in the LHC.

For the analysis of quenches in large size, forced flow cooled superconducting magnets for fusion, computer codes for the simulation of the quench process have been widely used (1). For accelerator magnets, the existing codes (2) (3) describe well some aspects of the quench process, but a code for realistic simulations of quenches in complex systems is not available.

The program $S P Q R$ (Simulation Program for $Q$ uench Research) has been developed to study the thermodynamic and electro-magnetic processes during a quench by solving the heat balance equation focusing on the particularity of the quench processes in accelerators magnets and busbars. It is of particular interest to calculate the quench propagation velocity along the superconductor, and between adjacent turns in a magnet coil. Section 2 introduces the theoretical model. An overview over the results of simulation studies that were performed with $S P Q R$ and their comparison with experimental data are presented in the Sections 3 and 4. Results for the temperature profiles after a quench in a superconducting busbar at the LHC obtained with an earlier numerical model were already published (4).

\section{$2 \quad$ Modelling}

\subsection{General remarks}

To evaluate the quench propagation, the computation starts by initialising a quenched zone as a Gaussian like temperature distribution along the superconductor that carries a current $\mathrm{I}(\mathrm{t})$. Bath temperature and the parameters of the superconducting cable and the insulation need to be specified.

The temperature profile is then evaluated as a function of time and space approximating the heat balance equation with the finite difference method, taking into account the longitudinal heat flux $k(T) d T / d x$ along the conductor, the thermal impedance $c(T) d T / d t$ and the heat generation $G(T)$ in the 
conductor, and the transverse cooling $h^{H e}(T) P . P$ is called the wetted perimeter, which is $P=f \cdot 2 \pi r$ for a circular conductor, $r$ being the radius of the conductor and $f$ being the effective fraction. Several transverse cooling models are included: direct heat transfer from the surface of the conductor into the helium bath (with the heat transfer coefficient $h^{H e}(T, d T / d t)$, heat transfer through an insulation layer into the helium, or assuming a certain helium fraction per unit length inside the cable to make the simulation more realistic and to fit experimental results. $P$ corresponds to the surface per unit length of the conductor that is surrounded by liquid helium. The simulation model assumes a homogeneous conductor, i.e., the material parameters are calculated according to the fractions of copper and superconductor and taking into account their temperature dependence.

\subsection{One-dimensional model}

The one-dimensional heat balance equation allows studying the quench propagation along a busbar and the forced quenching by heaters. It is given by

$$
\begin{aligned}
& \frac{d}{d x}\left(k(T(x, t)) \frac{d T(x, t)}{d x} A(x)\right)-h^{H e}(T(x, t), t) P(x)+\dot{\mathrm{q}}+G(T(x, t), t) \\
& =c(T(x, t)) A(x) \frac{d T(x, t)}{d t} .
\end{aligned}
$$

The term $\dot{q}$ stands for the initial heat pulse that triggers a quench. The impact of changing copper stabilisation and cooling conditions can be included in Eq. 1 as the conductor cross-section $A(x)$ and the cooling conditions $h^{H e}(T(x, t), t) P(x)$ are functions of the longitudinal position along the conductor.

The heat generation $\mathrm{G}(\mathrm{T}(\mathrm{x}, \mathrm{t})$ in Eq. 1 is given by

$$
G= \begin{cases}0 & \text { if } T(x, t) \leq T_{c s}(t) \\ \rho_{c u}(T(x, t)) \frac{I(t)^{2}}{A_{c u}(x)} \frac{T(x, t)-T_{c s}(t)}{T_{c r i t}(t)-T_{c s}(t)} & \text { if } T_{c s}(t)<T(x, t) \leq T_{c r i t}(t) \\ \rho_{c u}(T(x, t)) \frac{I(t)^{2}}{A_{c u}(x)} & \text { if } T(x, t)>T_{c r i t}(t)\end{cases}
$$

with $\rho_{c u}$ being the copper resistivity, $A_{c u}$ the copper cross-section and $I$ the excitation current. The heat generation is zero below the current sharing temperature $T_{c s}$ when the conductor is still superconducting. A linear model of the current sharing is assumed so that the heat generation increases linearly beyond $T_{c s}$ until the critical temperature $T_{c r i t}$ is reached and the current flows only in the copper of the conductor. The normal conducting part of the cable is most of the time in this third regime during the quench process. 


\subsection{Heat transfer through an insulation layer}

The heat conductivity of the insulation material limits the amount of heat that is transported into helium. An adequate modelling of the heat transfer through an insulation layer requires a two-dimensional model. Solving the twodimensional heat balance equation in each time step with the finite difference method requires a significant amount of computational time. For that reason two different approaches are followed. Eq. 1 can be generalised as

$$
\begin{aligned}
& \frac{d}{d x}\left(A(x) j_{x}\right)+G\left((T(x, t), t)+\dot{\mathrm{q}}+F_{t}\left(T(x, t), \frac{d T(x, t)}{d t}\right)\right. \\
& =c(T(x, t)) A(x) \frac{d T(x, t)}{d t}
\end{aligned}
$$

$F_{t}$ is the transverse heat flux from the surface of the superconducting cable, which is a function of the temperature and its time derivative because of the thermal impedance of the insulation layer.

If the thickness of the insulation layer $l_{i s o}$ is small with respect to the diameter of the conductor, a linear temperature profile inside the insulation material is assumed. This reduces Eq. 3 to

$$
\begin{aligned}
& \frac{d}{d x}\left(k(T(x, t)) \frac{d T(x, t)}{d x}\right) A(x)+\dot{\mathrm{q}}+G(T(x, t), t) \\
& +k_{\text {iso }}\left(\frac{T(x, t)+T_{\text {iso }}(x, t)}{2}\right) P(x) \frac{T_{\text {iso }}(x, t)-T(x, t)}{l_{\text {iso }}} \\
& =c(T(x, t)) A(x) \frac{d T(x, t)}{d t}
\end{aligned}
$$

$T_{i s o}$ refers to the temperature on the surface of the insulation that is exposed to the helium bath. It is set to $T_{b a t h}$ at the start of the simulation and further evaluated as

$$
\begin{aligned}
& k_{\text {iso }}\left(\frac{T(x, t)+T_{\text {iso }}(x, t)}{2}\right) P(x) \frac{T(x, t)-T_{\text {iso }}(x, t)}{l_{\text {iso }}}-h^{H e}\left(T_{i s o}(x, t), t\right) P(x) \\
& =c_{\text {iso }}\left(T_{i s o}(x, t)\right) A(x) \frac{d T_{i s o}(x, t)}{d t}
\end{aligned}
$$

A precise evaluation for the transverse heat transfer requires to discretise the radial heat balance equation and to solve it for every time step (so-called time transient). Neglecting the longitudinal heat propagation in the insulation 
layer with respect to that of the superconducting structure, the heat balance equation for the insulation layer is

$$
-\vec{\nabla} \cdot \vec{j}(r, \varphi)=c_{i s o} \cdot \frac{d T_{i s o}}{d t}
$$

Assuming radial symmetry, Eq. 6 changes to $-(d / r d r)\left(r \cdot k_{i s o} \cdot(d T / d r)\right)=$ $c_{i s o} \cdot\left(d T_{i s o} / d t\right)$. In this case the evaluation of $F_{t}(T, d T / d t)$ is carried out before the simulation of the quench propagation in the conductor. The first computation starts with a homogeneous temperature profile at $T_{\text {bath }}$ inside the insulation layer. The temperature of the superconducting wire is increased with a constant $d T / d t$ and the heat flux is evaluated for each temperature step. This procedure is repeated for various rates of $d T / d t$ and the results are stored in a two-dimensional array as function of $T$ and $d T / d t$. It follows the temperature evaluation of the conductor. The stored values are read and linearly interpolated to receive the correct values of $F_{t}(T, d T / d t)(5)$.

\subsection{Heat Transfer into Helium}

Two different models can be used to determine the heat transfer into the helium bath (taken from P. Bauer (6)). The simple model (model 1) for the heat transfer coefficient $h$ is

$$
h_{s}^{H e}(T(x, t), t)=a \cdot\left(T(x, t)-T_{b a t h}\right)
$$

The complex model (model 2) for transient heat transfer including Kapitza regime and film boiling requires more parameters

$$
h^{H e}= \begin{cases}0 & \text { if } T(x, t) \leq T_{b a t h} \\ a_{1} \cdot\left(T(x, t)^{n}-T_{b a t h}^{n}\right) & \text { if } T(x, t) \leq T_{n b} \\ a_{2} & \text { if } T_{f b}>T(x, t)>T_{n b} \\ a_{3} \cdot\left(T(x, t)^{m}-T_{b a t h}^{m}\right) & \text { if } T(x, t)>T_{f b} \wedge t-t\left(T>T_{n b}\right)<t_{f b} \\ a_{4} & \text { if } t-t\left(T>T_{f b}\right)>t_{f b}\end{cases}
$$

with $T_{n b}$ and $T_{f b}$ being the temperatures of starting nucleate and film boiling, $t_{f b}$ being the time interval for burn-out, and $a_{i}, n, m$ being the material and surface dependent constants (7). These constants vary for the simulation of heat transfer from the conductor into helium and from the surface of the insulation layer into cooling bath. 


\subsection{Three-dimensional model}

The three-dimensional heat balance equation is to be solved in order to study the transverse quench propagation in between neighbouring turns. The average conductor height $D y$ and its width $D z$ are used for the discretisation in $y$ and $z$. A constant temperature is assumed within a volume element $d V=D x \cdot D y \cdot D z$. In this model the conductor cross-section is a constant $A(x) \equiv A=D y \cdot D z$. The heat transfer between two turns is given by the heat conduction through the insulation between two turns, using the thermal conductivity $k_{i s o}^{t}$ and the insulation layer thickness $h_{i s o}^{t}$. The three-dimensional heat balance equation is given by

$$
\begin{aligned}
& k(T(x, y, z, t))\left(\frac{d^{2} T(x, y, z, t)}{d x^{2}}+\frac{d^{2} T(x, y, z, t)}{d y^{2}}\right)+\frac{\dot{\mathrm{q}}}{A(x, y, z)}+ \\
& \frac{k_{i s o}^{t}(T(x, y, z, t))}{l_{i s o}^{t}} \frac{d T(x, y, z, t)}{d z}-h^{H e}(T(x, y, z, t), t) \frac{P(x, y)}{A(x, y, z)}+ \\
& g(x, y, z) k_{i s o}^{h}\left(\frac{T(x, y, z, t)+T_{h}(t)}{2}\right) \frac{w_{t}}{l_{i s o}^{h} A(x, y, z)}\left(T_{h}(t)-T(x, y, z, t)\right)+ \\
& \frac{G(T(x, y, z, t), t)}{A(x, y, z)}=c(T(x, y, z, t)) \frac{d T(x, y, z, t)}{d t}
\end{aligned}
$$

\section{Protection by Quench Heaters}

To avoid overheating of the superconducting cable, quench heater strips are installed in the LHC main dipole and quadrupole magnets, in the high field region between coils and collars (high field heaters strips, HF) and in the low field region (low field heater strips, LF). After the detection of a quench, a capacitor with the capacitance $C_{h}$ is discharged into the heater strip made out of stainless steel with a resistance $R_{h}$ to distribute the stored energy across the coils. The temperature of the initial quenched zone (hot spot temperature), depends strongly on the heater delay that is the time between the start of the discharge and the heater induced quench. As an example, for the dipole magnets operating at full current, an additional heater delay of $10 \mathrm{~ms}$ leads to an increased hot spot temperature of 30-40 K.

The heater delay depends on the layout of the strips, the parameters for the discharge, the parameters of the superconductor, the insulation between strips and conductor, and the excitation current. The delay can be estimated by calculating the heat flux through the insulation into the superconductor. The heat balance equation for the heater strip is 


$$
\begin{aligned}
& \frac{\rho_{h}\left(T_{h}(t)\right) \cdot I_{h}^{2}(t)}{A_{h}}-k_{i s o}^{h}\left(\frac{T(x, t)+T_{h}(t)}{2}\right) \frac{w_{h}}{l_{i s o}} \cdot f_{h} \cdot\left(T_{h}(t)-T(x, t)\right) \\
& =c_{h}\left(T_{h}\right) \cdot A_{h} \cdot \frac{d T_{h}(t)}{d t}
\end{aligned}
$$

The index $h$ refers to the material properties of the heater strip. The heat transfer through the insulation into the superconducting cable is given by the thermal conductivity of the insulation material $k_{i s o}^{h}(T)$, the heater strip width $w_{h}$ which corresponds to the surface per unit length and the insulation length $l_{\text {iso. }}$. The model assumes a linear temperature gradient in the insulation layer between the heater strip and the conductor. For that reason the average temperature $\left.\left(T(x, t)+T_{h}(t)\right) / 2\right)$ is used to evaluate the heat flux from the strip through the insulation into the conductor. The factor $f_{h}$ in Eq. 10 has been introduced to adjust the effective fraction of heat which is conducted from the heater strip into the superconducting cable.

The heat generation in the heater strip is calculated with a constant capacitance $C_{h}$ of the heater power supply. The initial heater current is $I_{h}^{0}=$ $U_{h}^{0} / R_{h}\left(T=T_{b a t h}\right)$, with $U_{h}^{0}$ the loading voltage and $R_{h}\left(T=T_{b a t h}\right)$ the heater resistance at bath temperature. The temperature dependent heater strip resistance is evaluated with the new temperature after each time-step.

It is sufficient to heat some sections of the superconducting cable as the quench propagates longitudinally into the non-heated sections with typical velocities in the range of $15 \mathrm{~m} / \mathrm{s}$ to $20 \mathrm{~m} / \mathrm{s}$ at nominal current of about $11.8 \mathrm{kA}$ for the LHC main dipole magnets (8). As an example, a $400 \mathrm{~mm}$ long section would be quenched in less than $10 \mathrm{~ms}$. Therefore, the austenitic stainless steel strips are partially plated with copper. The reduced resistance allows connecting two heater strips in series, thus reducing the number of heater power supplies by a factor of two. The quench heater design is shown in Fig. 1.

The heater resistance is $R_{h}(T(t))=\left(\rho_{h}(T(t)) l_{h}\right) / A_{h} \cdot\left(l_{h}^{F e}\right) /\left(l_{h}^{F e}+l_{h}^{C u}\right)$, with $l_{h}$ being the total length of the heater strip, $l_{h}^{F e}$ being the heated part of the strip, and $l_{h}^{C u}$ being the copper plated part. The current through the heater strip is given by:

$$
I_{h}(t)= \begin{cases}0 & \text { if } t<t_{\text {det }} \\ I_{h}^{0} \cdot \exp \left(-\frac{t}{R_{h}(T(t)) C_{h}}\right) & \text { if } t \geq t_{\text {det }}\end{cases}
$$

A simulation study was performed to optimise the copper plating cycle and the insulation layer thickness between heater strip and coil in order to minimise the quench heater delays at low and high magnet current.

The quench heaters strips are $0.025 \mathrm{~mm}$ thick and $15.0 \mathrm{~mm}$ wide, glued be- 


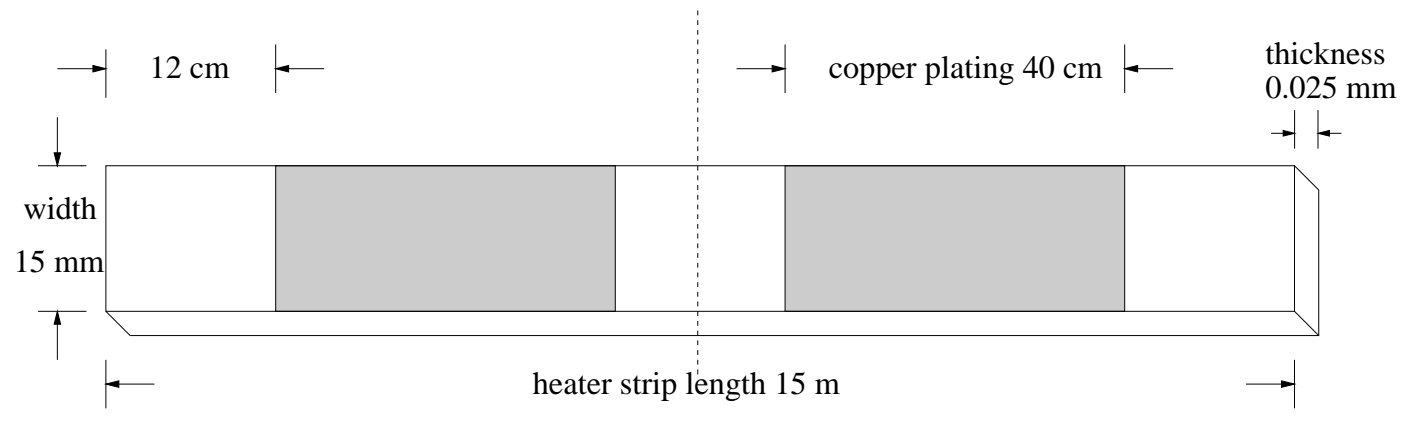

Fig. 1. Heater strip design.

tween two layers of polyimide electrical insulation foil of $0.075 \mathrm{~mm}$ thickness each (see Fig. 1). Two strips are connected in series $\left(l_{h}=30 \mathrm{~m}\right)$. The capacitor discharge supply with a capacitance of $7.05 \mathrm{mF}$ has a maximum voltage of $900 \mathrm{~V}$. Since the lifetime of capacitors increase with decreasing voltage, an operating voltage of $750 \mathrm{~V}$ was assumed.

Two parameters are analysed: 1) The "initial" heater delay is defined as the time in between firing the heaters and provoking a quench in the conductor covered by a heated part of the heater strip. This delay becomes shorter by increasing the copper plated length which reduces the heater strip resistance and increases the initial current. 2) The "total" heater delay, which is the time in between firing the heaters and having provoked a quench in the entire conductor under the heater strip.

The simulation model was cross-checked by comparing the simulated quench propagation velocity with experimental results (about $15-20 \mathrm{~m} / \mathrm{s}$ at nominal current). A typical example of the simulation output is given in Fig. 2, which shows the simulated temperature profiles along the cable for various times including the provoked quenching by the non-copper plated parts of the heater strips.

In order to compare the heater delays for different copper cladding at low current, adiabatic conditions are assumed to exclude the uncertainty in the long time behaviour of the cooling model by heat transfer into helium. In a second step the simulations are repeated including the cooling. The results for the dipole magnet operating at injection current of $750 \mathrm{~A}$ demonstrated that longer heated parts yield shorter heater delays at low current. This is due to the minimum propagation zone. If only the minimum quench energy $(M Q E)$ is dissipated into the conductor the minimum propagation zone $(M P Z)$ exceeds $20 \mathrm{~cm}$ at injection current. As the energy dissipated into the superconducting cable by the heaters is significantly higher than $M Q E$ the heated part can be shorter. A heated length of $8 \mathrm{~cm}$ gives shortest initial heater delay assuming adiabatic conditions. Including heat transfer into helium a heated zone of less than $8 \mathrm{~cm}$ is not sufficient to provoke a quench at injection current when the initial heater voltage was limited to $900 \mathrm{~V}$. 


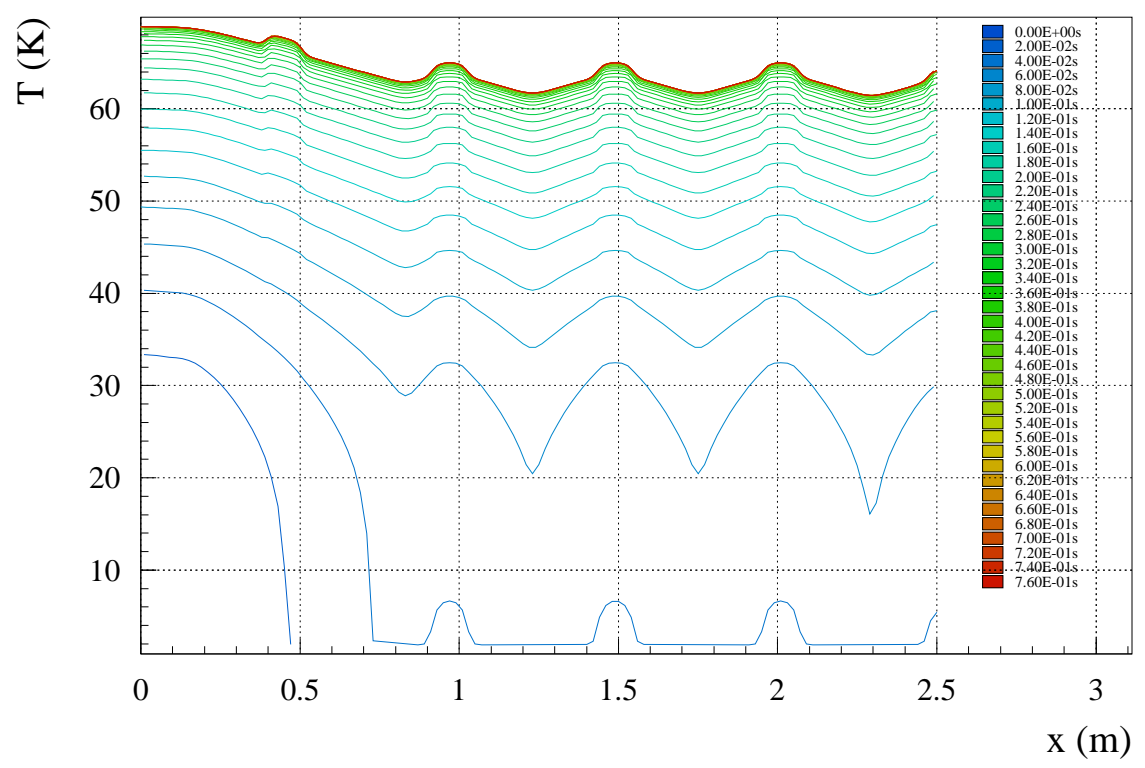

Fig. 2. Simulation of the temperature profile along the cable with forced quenching by copper plated heaters. The time interval between two plotted curves is $20 \mathrm{~ms}$. The current was $12.8 \mathrm{kA}$, cable cross section $19.2 \mathrm{~mm}^{2}, 3 \%$ helium content, $r_{C u / S c}=1.9$, $\mathrm{RRR}=100$, initial time step for the computation $0.05 \mu \mathrm{s}$.

The results from the simulation for "initial" and "total" heater delays at nominal current are summarised in Fig. 3. Combining the results at injection and nominal current a heated length of $10-12 \mathrm{~cm}$ and a copper plating length of $40-50 \mathrm{~cm}$ gives the best performance for the entire operational range. For manufacturing purposes a heated strip design consisting of a cycle of $12 \mathrm{~cm}$ heated part and $40 \mathrm{~cm}$ copper plated part was chosen to simplify the series production of $15 \mathrm{~m}$ long heater strips.

The "initial" heater delays from simulation and experiments are shown in Table 1.

Table 1

\begin{tabular}{c|c|c|c|c|c|}
$\begin{array}{c}\text { Pattern } \\
\text { Fe-Cu }[\mathrm{mm}]\end{array}$ & $\mathrm{P} 0 / \mathrm{A}$ & $\tau$ & $U_{\min }$ & \multicolumn{2}{|c}{ Heater delay $[\mathrm{ms}]$} \\
{$\left[\mathrm{W} / \mathrm{cm}^{2}\right]$} & {$[\mathrm{ms}]$} & {$[\mathrm{V}]$} & measured & simulation \\
\hline $250-250$ & 50 & 85 & 750 & 30 & 34 \\
$120-240$ & 35 & 112 & 900 & 35 & 38 \\
$120-360$ & 60 & 85 & 750 & 28 & 30 \\
$120-400$ & 70 & 77 & 700 & 25 & 28 \\
$120-480$ & 94 & 68 & 700 & - & 24 \\
$100-400$ & 94 & 68 & 750 & - & 26 \\
$40-240$ & 112 & 48 & $\geq 900$ & 25 & 30
\end{tabular}

Comparison of measured and predicted quench heater delays. The simulations were carried out for the HF heaters only. P0/A is the initial power density, and $\tau$ the time constant for the heater pulse. 


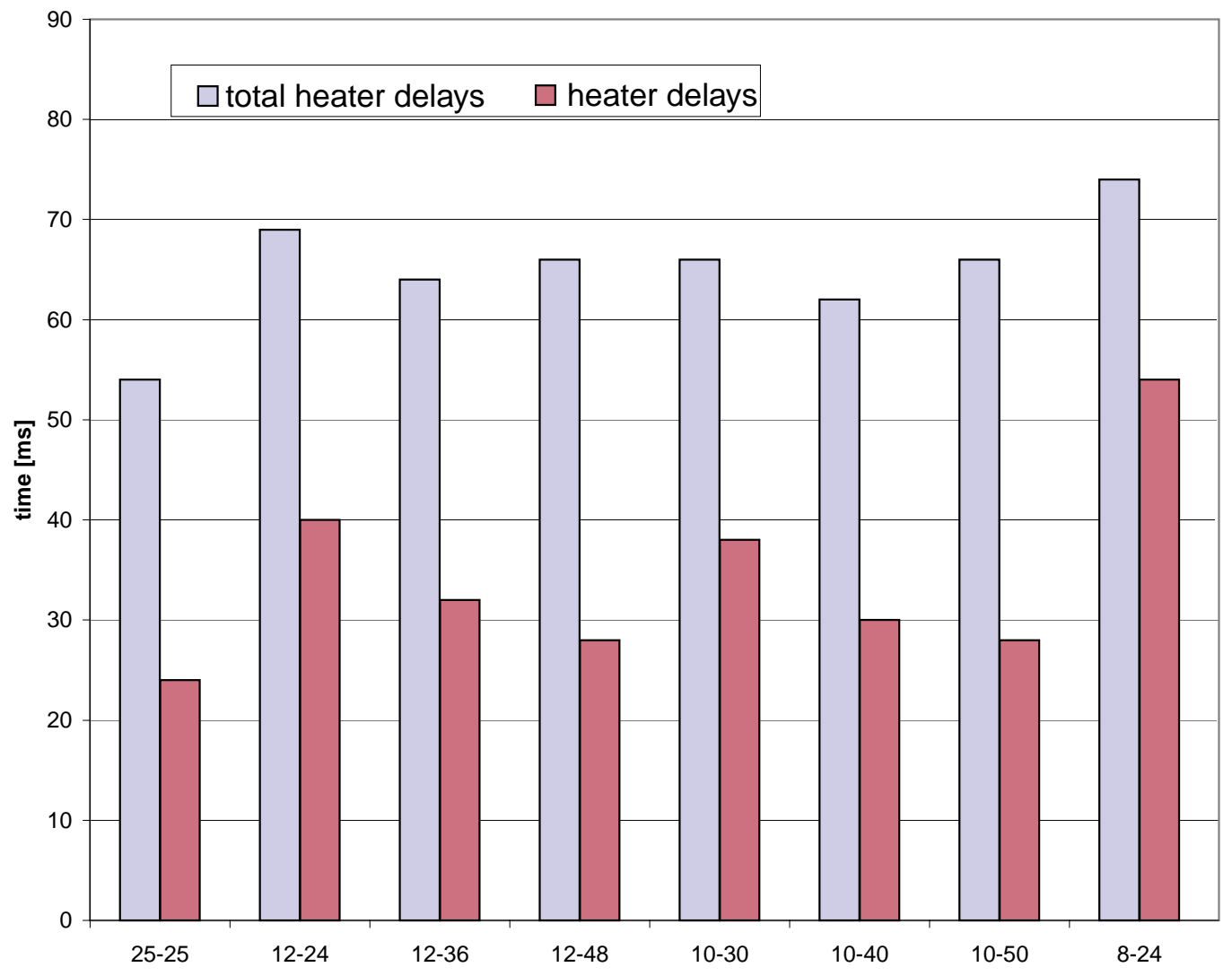

Fig. 3. Heater delays from a simulation at nominal current as a function of the copper plating ratio (heated/non heated lengths in $\mathrm{cm}$ ). The energy required for the plating ratio of $12-48$ is less than a factor of two compared to a ratio of $25-25$, the increase of heater delay is acceptable.

The simulation studies show an exponential increase of the heater delays as a function of the thickness of the insulation layer. A strong increase of the heater delay has been seen for tests on a short dipole magnet with an increased insulation layer thickness. For redundancy, it is required to protect the magnet with only half of the high field heaters. Therefore an increase of the insulation layer thickness by a factor of two is not permissible using the same type of polyimide film that is foreseen for the insulation of LHC cables. The change to another insulation material with higher thermal conductivity would of course allow an increased thickness of the insulation layer. 


\section{Magnetic quench back}

\subsection{Motivation}

During the development programme for the LHC many different types of prototype magnets were tested. Current decay and voltage across parts of the magnets, such a poles or blocks, were recorded during training quenches and quenches initiated with heaters. Here observations for two of the magnets are described: During the test of a sextupole magnet one pole quenched and the current decayed from $600 \mathrm{~A}$ with a time constant in the order of $100 \mathrm{~ms}$ (9). An inductive voltage was observed across the non-quenching poles for about $60 \mathrm{~ms}$, showing that only one pole started to quench. After about $60 \mathrm{~ms}$ all other poles quenched. For a magnet with impregnated coils a quench propagation along the wire to all other coils at the same time is not possible, but this observation can be well explained with quench back.

In an experiment with a $10 \mathrm{~m}$ long prototype dipole magnet, only the heaters installed on the coils in one aperture of the twin aperture magnets were fired (10). The current in the magnet decayed with a time constant of about $150 \mathrm{~ms}$. The inductive voltage across the coil in a non-quenching aperture would exceed $1 \mathrm{kV}$. In the experiments it was observed that the voltage did not exceed $100 \mathrm{~V}$, which is explained by a fast quench of the second aperture. A thermo-hydraulic quench propagation is unlikely, since the time constant of quench propagation via warm helium is several seconds (19). When quench back is included in the simulation the observations can be understood.

Following M.A.Green (11), quench back in superconducting magnets has two causes. Thermal quench back: normal regions can be induced by heat transfer from components outside the coil to the superconductor. Thermal quench back is used in superconducting solenoid magnets as an integral part of the magnet quench protection system (12). Magnetic quench back: normal regions can be induced by $\mathrm{AC}$ losses from eddy currents in the superconductor due to changing magnetic field. Eddy currents and AC losses have been investigated to understand field errors during the current ramp, when eddy currents dominate the field errors in accelerator magnets. Interstrand coupling currents in Rutherford type cables were modelled by means of a discrete network of nodes in (13). AC losses from interfilament coupling currents have been investigated in (14). The impact of interfilament coupling currents for protection of multicoil magnets has been studied in (15).

For the LHC main dipole and quadrupole magnets, after firing quench heaters, the excitation current decays with a time constant in the order of some hundred milliseconds, compared to the time for current ramping of about $20 \mathrm{~min}$. 
The losses from both interfilament and interstrand currents for a fast change of the magnetic field during a quench are some orders of magnitude larger than $\mathrm{AC}$ losses during the ramp. Heating power due to interstrand and interfilament coupling currents can quench large fractions of the magnet coils after some ten milliseconds. The superconductor would also quench when the sum of excitation current and induced current exceeds the critical current, but this has not been observed in the LHC magnets.

\subsection{Modelling}

In order to calculate the power of the induced coupling currents their built-up time that is characteristic for the dimensions of the filaments, the strands and the cable has to be considered. An additional contribution is due to possible magnetisation losses since a strong external magnetic fields can penetrate the filament and cause a magnetisation that leads to a hysteresis and an additional contribution to the loss power during a changing field strength $d / d t \int M(B) d B$.

The equations presented below are given by A. Verweij (13). The magnetisation is:

$$
M=-\frac{2}{3 \pi} J_{c} d_{f} \frac{d B / d t}{|d B / d t|}
$$

with $J_{c}=I_{c} / A_{N b T i}$ being the critical current density and $d_{f}$ being the filament diameter. The interfilament current is calculated with

$$
\begin{aligned}
I_{i f}(t) & =\frac{d B}{d t} \frac{L_{p_{f}}^{2} d_{f}}{4 \pi^{2} \rho(T(t))} \cdot\left(1-\exp \left(-\frac{t}{\tau_{i f}}\right)\right) \\
\tau_{i f} & =\frac{\mu_{0}}{2 \rho(T(t))}\left(\frac{L_{p_{f}}}{2 \pi}\right)^{2}
\end{aligned}
$$

with $\tau_{i f}$ being the time constant of the interfilament coupling and $L_{p_{f}}$ being the filament twist pitch. Both effects lead to the combined interfilament losses of coupling currents and magnetisation

$$
\begin{aligned}
P / V_{i f}= & \frac{1}{\rho}\left(\frac{d B}{d t}\right)^{2}\left(\frac{L_{p_{f}}}{2 \pi}\right)^{2} \cdot\left(1-\exp \left(-\frac{t}{\tau_{i f}}\right)\right)^{2} \\
& +\frac{2}{3 \pi} J_{c} d_{f}\left|\frac{d B}{d t}\right|\left(1-\exp \left(-\frac{t}{\tau_{i f}}\right)\right)
\end{aligned}
$$


The interstrand currents in a Rutherford cable have been computed numerically to be (13)

$$
\begin{aligned}
I_{s}= & \left(0.0415 \frac{L_{p_{s}} w N_{s, l}}{R_{c}} \frac{d B}{d t} \cos (\phi)+0.25 \frac{L_{p_{s}} h}{R_{a} N_{s, l}} \frac{d B}{d t} \sin (\phi)\right) \\
& \left(1-\exp \left(-\frac{t}{\tau_{i s}}\right)\right) ; \\
\tau_{i s}= & 1.68 \cdot 10^{-8} \frac{L_{p_{s}}\left(N_{s, l}^{2}-4 N_{s, l}\right)}{R_{c}}
\end{aligned}
$$

with $L_{p_{s}}$ being the strand twist pitch, $N_{s, l}$ the number of strands per layer, $w$ the width and $h$ the height of the strand, $R_{a}$ the contact resistance in between adjacent strands in one layer and $R_{c}$ being the cross resistance in between the two layers. The angle $\phi$ determines the parallel and rectangular component of the magnetic field with respect to the broad side of the cable (a perpendicular field component has $\phi=0$, a parallel field component has $\phi=\pi / 2$ ) The interstrand losses are given by

$$
\begin{aligned}
P / V_{i s}= & \left(0.170 \frac{L_{p_{s}} w^{2}\left(1-1 / N_{s, l}\right)}{R_{a}}(\cos (\phi))^{2}+0.125 \frac{L_{p_{s}} h^{2}}{R_{a}}(\sin (\phi))^{2}+\right. \\
& \left.8.49 \cdot 10^{-3} \frac{L_{p_{s}} w^{2}\left(N_{s, l}^{2}-N_{s, l}\right)}{R_{c}}(\cos (\phi))^{2}\right) \\
& \left(\frac{d B}{d t}\right)^{2}\left(1-\exp \left(-\frac{t}{\tau_{i s}}\right)\right)^{2}
\end{aligned}
$$

For the simulation of the impact of eddy currents, the magnetic field is adjusted at every time step as a function of the current $B(I(t))=a+b I(t)$ and the time derivative is computed. The resistivity, and other parameters of interest are evaluated at every time step and element.

\subsection{Experiments with $\mathrm{LHC}$ dipole magnets}

The starting time of the quench back depends on the amplitude of the magnetic field, its angle with respect to the broad side of the cable, the value of the contact resistances $\left(R_{a}, R_{c}\right.$ for interstrand coupling currents and the effective copper matrix inside a strand for the interfilament coupling currents), the eddy current time constant and $d B / d t$. The field components parallel and perpendicular to the broad side of the cable of the LHC dipole magnet are shown in Fig 4.

An example for a quench of a single aperture short dipole magnet showing the voltages across the blocks is given in Fig. 5. The quench was provoked by 


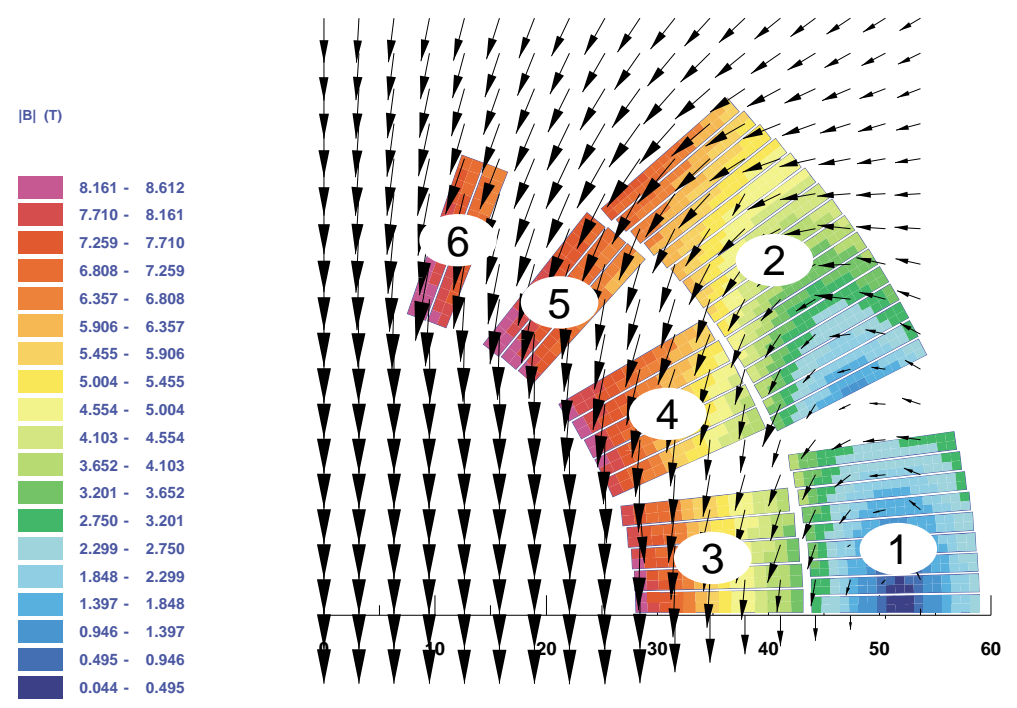

Fig. 4. LHC dipole magnet $2 \mathrm{~d}$ cross-section showing the magnetic field map (calculated at nominal field with ROXIE 8.0 (16))

heater strips in the outer layer. The quench starts in block 2 of the lower pole and propagates to block 6 and 5 (left part of Fig. 5). Due to the current decay the voltage across blocks that did not quench is inductive. At about $18 \mathrm{~ms}$ the block voltages start to become partly resistive. This becomes more clear in the right part of Fig. 5, in which the inductive voltages have been subtracted. The quench back starts first in block 4 . The quench back starting time depends mainly on the field component perpendicular to the broad cable side, and the voltage rise after the quench back start is dominated by the magnitude of the magnetic field due to magneto-resistance.
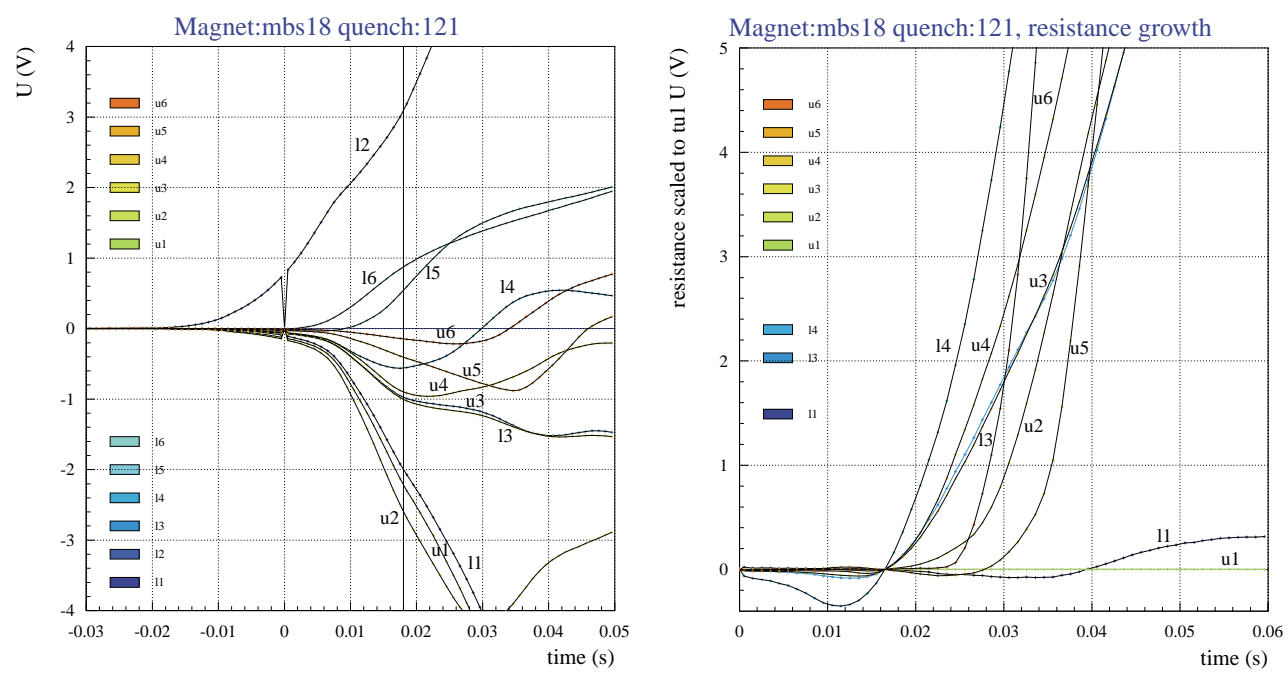

Fig. 5. Quench back example: block voltages of a short dipole magnet after a quench (left); the same voltages taking out the inductive voltage (right). 
A parameter study was carried out to determine the quench back starting time $t_{Q B}$ as a function of the time constant for the current decay, values of $R_{c}$ and $R_{a}$, the initial current and various positions in the LHC dipole magnet (see Table 2). The current decay was approximated by a Gaussian function with the time decay constant $\tau$.

Table 2

\begin{tabular}{c|c|c|c|c} 
block & $\begin{array}{c}B_{\perp} \\
{[\mathrm{T}]}\end{array}$ & $\begin{array}{c}B_{\|} \\
{[\mathrm{T}]}\end{array}$ & $\begin{array}{c}|B| \mathrm{T}] \\
{[\mathrm{rad}]}\end{array}$ & $\begin{array}{c}\phi \\
{[\mathrm{rad}}\end{array}$ \\
\hline 1 & -0.838 & -1.332 & 1.57 & 1.01 \\
2 & -0.820 & -4.058 & 4.14 & 1.37 \\
3 & -6.006 & -0.900 & 6.07 & 0.15 \\
4 & -5.366 & -3.700 & 6.52 & 0.60 \\
5 & -3.980 & -6.254 & 7.41 & 1.00 \\
6 & -1.820 & -7.568 & 7.78 & 1.33
\end{tabular}

The average magnetic field in the conductor blocks and their parallel and perpendicular to the blocks. The angle is defined such that 0 means a pure perpendicular, $\pi / 2$ means pure parallel field with respect to the broad side of the cable.

In some experiments with various short and long prototype dipole magnets the number of heater strips that are fired was changed, since this affects the resistance growth and therefore the current decay time constant $\tau$. The simulation results for the quench back time $t_{Q B}$ are in good agreement with experimental results (see Table 3). The table does not include data for the outer layer blocks as quenches are induced there with heaters. The impact of quench back on the magnet protection depends on its starting time. The simulated values for quench back starting times range from $30 \mathrm{~ms}$ for $\tau=0.2 \mathrm{~s}$ to $60 \mathrm{~ms}$ for $\tau=0.3 \mathrm{~s}$ at nominal current and average field in block 3 . Without provoking a forced quench by heaters, the current decay is too slow to induce significant magnetic quench back. Firing one heater strip per pole already induces quench back fast enough to avoid magnet degradation (8). The minimum current at which quench back occurs is computed to be about $5 \mathrm{kA}$. During the experiments quench back occurred for quenches at $6 \mathrm{kA}$ whereas at $3 \mathrm{kA}$ no quench back was observed.

Further simulations were carried out to study the influence of $R_{c}$ and $R_{a}$. The results are summarised in Table 4 . The typical contact resistance values of $R_{c}=20 \mu \Omega$ and $R_{a}=100-150 \mu \Omega$ for the dipole Rutherford cables were taken from ramp rate sensitivity measurements (18). Assuming these values the interstrand coupling losses are mainly induced by the field component perpendicular to the broad side of the cable over the contact resistances $R_{c}$.

For Block 3 the dominating quench mechanism are interstrand coupling currents. For Block 6 the magnetic field is almost parallel to the broad side of the cable, and losses due to interfilament currents $P_{\text {if }}$ dominate. When the values for $R_{c}$ and $R_{a}$ are increased, the quench back is in general dominated by in- 


\begin{tabular}{l|c|c|c|c|c|c|c|c|c|c} 
Quench & Block & $I_{0}$ & $\tau$ & $B$ & angle & $R_{c}$ & $R_{a}$ & $A_{C u}$ & $t_{Q B}^{\text {sim }}$ & $t_{Q B}^{\text {exp }}$ \\
{$[k A]$} & {$[m s]$} & {$[T]$} & rad & {$[\mu \Omega]$} & {$[\mu \Omega]$} & {$\left[m^{2}\right]$} & {$[m s]$} & {$[m s]$} \\
\hline 1 & 3 & 11.7 & 203 & 6.07 & 0.148 & 20 & 100 & 15.4 & 32 & $30 \pm 6$ \\
1 & 4 & 11.7 & 203 & 6.51 & 0.603 & 20 & 100 & 15.4 & 35 & $35 \pm 5$ \\
1 & 5 & 11.7 & 203 & 7.41 & 1.004 & 20 & 100 & 15.4 & 39 & $40 \pm 4$ \\
1 & 6 & 11.7 & 203 & 7.78 & 1.334 & 20 & 100 & 15.4 & 40 & $42 \pm 3$ \\
\hline 2 & 3 & 11.7 & 178 & 6.07 & 0.148 & 20 & 100 & 15.4 & 27 & $25 \pm 5$ \\
2 & 4 & 11.7 & 178 & 6.51 & 0.603 & 20 & 100 & 15.4 & 30 & $30 \pm 4$ \\
2 & 5 & 11.7 & 178 & 7.41 & 1.004 & 20 & 100 & 15.4 & 32 & $34 \pm 3$ \\
2 & 6 & 11.7 & 178 & 7.78 & 1.334 & 20 & 100 & 15.4 & 33 & $36 \pm 3$ \\
\hline 3 & 3 & 11.7 & 276 & 6.07 & 0.148 & 20 & 100 & 15.4 & 49 & $45 \pm 8$ \\
3 & 4 & 11.7 & 276 & 6.51 & 0.603 & 20 & 100 & 15.4 & 54 & $58 \pm 6$ \\
3 & 5 & 11.7 & 276 & 7.41 & 1.004 & 20 & 100 & 15.4 & 58 & $60 \pm 6$ \\
3 & 6 & 11.7 & 276 & 7.78 & 1.334 & 20 & 100 & 15.4 & 60 & $62 \pm 4$ \\
\hline 4 & 3 & 11.7 & 266 & 6.07 & 0.148 & 20 & 100 & 15.4 & 47 & $44 \pm 8$ \\
4 & 4 & 11.7 & 266 & 6.51 & 0.603 & 20 & 100 & 15.4 & 51 & $54 \pm 7$ \\
4 & 5 & 11.7 & 266 & 7.41 & 1.004 & 20 & 100 & 15.4 & 56 & $59 \pm 6$ \\
4 & 6 & 11.7 & 266 & 7.78 & 1.334 & 20 & 100 & 15.4 & 57 & $61 \pm 4$
\end{tabular}

Table 3

Example of the parameter study for quench back effect in main magnets (with Rutherford type cable) and the comparison of the simulation results with experimental data ( $1=$ training quench; $2,3,4$ quenches provoked with spot heaters; $2=$ protection with all HF heaters, $3=$ protection with half HF heaters, $4=$ protection with all LF heaters). The different values for $\tau$ are due to the number of heater strips fired. The quenches have been performed on the $15 \mathrm{~m}$ long prototype dipole magnet MBP2N1v3.

\begin{tabular}{l|c||c|c|c|c||c|c|c|c}
$\begin{array}{l}R_{a} \\
{[\mu \Omega]}\end{array}$ & $\begin{array}{c}R_{c} \\
{[\mu \Omega]}\end{array}$ & Block & $\begin{array}{c}t_{Q B}^{\text {sim }} \\
{[\mathrm{ms}]}\end{array}$ & $\begin{array}{c}P_{\text {if }} \\
{[\mathrm{W} / \mathrm{m}]}\end{array}$ & $\begin{array}{c}P_{\text {is }} \\
{[\mathrm{W} / \mathrm{m}]}\end{array}$ & Block & $\begin{array}{c}t_{Q B}^{\text {sim }} \\
{[\mathrm{ms}]}\end{array}$ & $\begin{array}{c}P_{\text {if }} \\
{[\mathrm{W} / \mathrm{m}]}\end{array}$ & $\begin{array}{c}P_{i s} \\
{[\mathrm{~W} / \mathrm{m}]}\end{array}$ \\
\hline 100 & 0.10 & 3 & 5.05 & 0.26 & 0.02 & 6 & 40.0 & 10.3 & 0.42 \\
100 & 1.00 & 3 & 37.1 & 12.2 & 62.5 & 6 & 56.5 & 14.8 & 9.19 \\
100 & 10.0 & 3 & 46.4 & 16.4 & 232 & 6 & 57.4 & 15.4 & 13.1 \\
100 & 50.0 & 3 & 47.4 & 18.2 & 65.4 & 6 & 57.4 & 15.4 & 3.02 \\
100 & 100 & 3 & 47.6 & 18.2 & 34.3 & 6 & 57.4 & 15.4 & 1.59 \\
1.00 & 20.0 & 3 & 47.0 & 16.9 & 432 & 6 & 57.4 & 15.4 & 23.8 \\
10.0 & 20.0 & 3 & 47.1 & 17.2 & 171 & 6 & 57.5 & 15.6 & 8.77 \\
50.0 & 20.0 & 3 & 47.1 & 17.3 & 148 & 6 & 57.5 & 15.6 & 7.43 \\
200 & 20.0 & 3 & 47.1 & 17.3 & 143 & 6 & 57.5 & 15.7 & 7.18
\end{tabular}

Table 4

Parameter study for quench back effect in main magnets magnets (with Rutherford type cable) with $I_{\text {init }}=11.7 \mathrm{kA}, \tau=0.26 \mathrm{~s}$ and $A_{C U}=15.4 \mathrm{~mm}^{2}$, values for the magnetic field from Table 2 , variation of $R_{c}$ and $R_{a}$.

terfilament coupling currents. For very small values of $R_{c}$ the model predicts strong induced coupling currents but little power dissipation. Adding these to the excitation current, the critical current could be exceeded.

Although the magnetic field and its angle with respect to the cable varies 
from about 0.5 Tesla and $90^{\circ}$ in Block 3 to about 9 Tesla in Block 6 and $5^{\circ}$, the quench back starting time scatter less than $15 \mathrm{~ms}$ for the different conductor blocks at nominal current, which is in agreement with experimental results.

Experiments and simulations demonstrate that quench back starts long before the time of maximum $d B / d t$ at about $0.2 \mathrm{~s}$, with $d B / d t \geq 20 \mathrm{~T} / \mathrm{s}$ and $d I / d t \geq 40 \mathrm{kA} / \mathrm{s}$ for the inner layer. With such gradient the coupling losses would be about $150 \pm 10 \mathrm{~W} / \mathrm{m}$ for the interfilament losses and $600 \pm 100 \mathrm{~W} / \mathrm{m}$ for the interstrand losses (with $R_{c}=20 \mu \Omega, R_{a}=100 \mu \Omega$, the values are given for Block 3 assuming an averaged magnetic field). At the start of the quench back typical values of $d B / d t$ are $8 \mathrm{~T} / \mathrm{s}$.

If the quench back starting time is small compared to $\tau_{i s}$, the following approximation can be made. As the time constant of the interstrand coupling currents $\left(\tau_{i s}\right)$ is about $\tau_{i s} \approx 25 \mathrm{~ms}$ for $R_{c}=20 \mu \Omega, R_{a}=100 \mu \Omega$ for the inner layer cable and $\tau_{i s}$ is proportional to $1 / R_{c}$, one can write

$$
1-\exp \left(-t / \tau_{i s}\right) \approx t / \tau_{i s} ; t \ll \tau_{i s}
$$

The interstrand coupling currents are proportional to $L_{p_{s}} / R_{c} \cdot(d B / d t)$ (see Eq. 18). Using the approximation of Eq. 19 the effect of $R_{c}$ cancels for times small compared to $\tau_{i s}$.

\subsection{Magnetic Quench Back in Corrector Magnets}

Magnetic quench back has been observed in many prototype corrector magnets (17). For only one magnet in the circuit, the current decays after a quench with a time constant of some ten milliseconds. Depending on the magnet and cable parameters, quench back can start as early as $10 \mathrm{~ms}$ after a natural or induced quench. The simulation results for magnetic quench back agree with results from tests with single corrector magnets. The program was used to extrapolate to the LHC configuration with up to 154 magnets powered in series. The results are shown in Tab. 5. The nominal current for most of the corrector magnets is $550 \mathrm{~A}$ and the inductance varies between a fraction of a $\mathrm{mH}$ and some ten $\mathrm{mH}$. The superconductor has a cross section of $0.61 \mathrm{~mm}^{2}$ and the ratio of $\mathrm{Cu} / \mathrm{NbTi}=1.9$. For the current decay time constant for the LHC, no magnetic quench back is expected.

The impact of the strand twist pitch of the conductor has been tested experimentally on two different MCS (spool piece sextupole magnets). A similar approximation as in Eq. 19 can be made which shows that the effect of the twist pitch lengths cancels for very short times of about $5 \mathrm{~ms}$ in a first order approach. Due to the significant different values of $R R R$ between the 
two magnets, the test could only be interpreted with the help of simulations, demonstrating that for a time less than the coupling current time constant, the resistivity has a more important impact than the twist pitch length on magnetic quench back.

\begin{tabular}{l|c|c|c|c|c|c|c|c}
$\mathrm{Lp}[\mathrm{m}]$ & 0.001 & 0.005 & 0.010 & 0.050 & 0.100 & 0.500 & 1.000 & 5.000 \\
\hline $\mathrm{RRR}=80, \tau=0.1 \mathrm{~s}$ & - & - & late & yes & yes & late & - & - \\
$\mathrm{RRR}=130, \tau=0.1 \mathrm{~s}$ & - & - & late & yes & yes & late & - & - \\
$\mathrm{RRR}=80, \tau=0.25 \mathrm{~s}$ & - & - & - & late & late & - & - & - \\
$\mathrm{RRR}=130, \tau=0.25 \mathrm{~s}$ & - & - & - & late & - & - & - & - \\
$\mathrm{RRR}=80, \tau=0.5 \mathrm{~s}$ & - & - & - & - & - & - & - & - \\
$\mathrm{RRR}=130, \tau=0.5 \mathrm{~s}$ & - & - & - & - & - & - & - & -
\end{tabular}

Table 5

Simulation study for quench back effect in corrector magnets. Lp is the twist pitch length of the filaments in the strand (for the wires of the LHC corrector magnets $\mathrm{Lp}=3 \mathrm{~cm})$

\section{Conclusions}

The simulation program SPQR is a tool that is being used in the optimisation process of the parameters for the protections system for the superconducting elements in the LHC, such as copper stabilisation of busbars, quench heater layouts, energy extraction devices, quench detection thresholds and more (22).

Since the simulation takes the cooling with helium through an insulation layer into account, the temperature profile along a busbar after a quench is in good agreement with experimental results (4). Studies are ongoing to better understand the quench process of small busbar cables emerged in superfluid helium that are routed through insulating plugs (23).

For the main dipole and quadrupole magnets, estimated heater delays due to the thermal conduction from heater strips through the insulation layer to the superconductor are in good agreement with the experimental data. The program was used for the optimisation of the copper plating cycle for the heater strips in the LHC main magnets.

Another application is to determine the time for magnetic quench back for dipole magnets and corrector magnets. The simulation results for the quench back starting time agree well with the experimental data from model dipole magnets and the first LHC 15-m prototype dipole magnet. This explains why quenches in such large magnets spread fast and no high voltage has been observed, even if only a part of the heater strips are fired. For corrector magnets, quench back depends on the inductance of the electrical circuit. During tests of single magnets, quench back is observed. In the LHC, most correctors will 
be powered in an electrical circuit comprising many magnets and no quench back is expected.

\section{Acknowledgement}

The authors wish to thank F.Rodriguez-Mateos, D.Hagedorn and A.Verweij for many fruitful discussion. The tests of the prototype magnets were performed by A.Siemko, L.Walckiers and their team. Without them kindly providing us with the test results, this paper could not have been written. M.Calvi and R.Herzog contributed with many ideas, in particular for the quench process of busbars, and provided us with an algorithm that became part of SQPR. Finally we like to acknowledge the comments from P.Bauer and L.Bottura.

\section{References}

[1] L. Bottura, PhD thesis, NET Report, Commission of the European Communities Directorate General XII - Fusion Programme, Brussels, 1993

[2] M. Wilson, Superconducting Magnets, OXFORD Science Publications, Oxford University Press, 1983

[3] D. Hagedorn and F. Rodriguez-Mateos, Modelling of the quench process in complex superconducting magnet systems, 12th Int.Conf. Magnet Technology, Leningrad, USSR, 23-28 June 1991

[4] F. Sonnemann, R. Schmidt, Modelling of the quench process for the optimisation of the design and protection of superconducting busbars for the LHC, ICEC 18, Bombay, India, 21-25 February 2000, India

[5] M. Calvi and R. Herzog provided the subroutine for this calculation

[6] P. Bauer, Stability of Superconducting Strands for Accelerator Magnets, PhD Thesis, Technical University of Vienna, 1999

[7] S.W. Van Sciver in Cryogenics 1998, Volume 38, page 503-512, Forced flow in He II cooling for superconducting magnets - design considerations

[8] F. Rodriguez-Mateos et al., Quench Heater Experiments on the LHC Main Superconducting Magnets, Proceedings of EPAC 2000

[9] R. Schmidt, A.Siemko, A.Ijspeert, Quench studies for the LHC corrector magnet MSBH, LHC Project Note 28, December 1995

[10] R. Schmidt, A. Siemko, F. Sonnemann, Quench process and Protection of the LHC Dipole Magnets, LHC Project Note 184, July 1999

[11] M.A. Green, Quench Back in thin Superconducting Solenoid Magnets, Cryogenics 24 (1984), pp.3-10

[12] M.A. Green, The Role of Quench Back in Quench Protection of a Superconducting Solenoid, Cryogenics 24 (1984), pp.659-667 
[13] A. Verweij, Electrodynamics of Superconducting Cables in Accelerator Magnets, PhD thesis 1995, University of Twente, Netherlands

[14] G.H. Morgan, Theoretical Behaviour of Twisted Multicore Superconducting Wires in a Time Varying Uniform Magnetic Field, J.Appl.Phys. 41 (1970), pp. 3673-3679

[15] K. Takeuchi et al., Interfilament coupling loss for protection of superconducting multicoil magnets, Cryogenics 38 (1998), pp.367-376

[16] S. Russenschuck et al., ROXIE: Routine for the Optimisation of Magnet X-Sections, CERN yellow report (1999)

[17] R. Schmidt et al., Protection of the superconducting corrector magnets for the LHC, EPAC 2000

[18] Z. Ang et al., Measurement of AC loss and magnetic field during ramp in the LHV model dipole, IEEE Trans.Appl.Supercond. 9 (1999), 742-745

[19] F. Rodriguez-Mateos, R. Schmidt, L. Serio, Thermo-hydraulic Quench Propagation at the LHC Superconducting Magnet String, ICEC 17, Bournemouth, UK, July 1998

[20] J.L. Perinet-Maquet et al., Measurement of superconducting busbars models for the LHC main dipole, Proceedings of MT16 1999

[21] R. Herzog et al., The superconducting busbar cable powering the auxiliary magnets of the LHC, Proceedings of CEC/ICM 1999

[22] K. Dahlerup-Petersen, The Protection System for the superconducting elements of the Large Hadron Collider, PAC 1999, New York City, USA, 29 Mar - 2 Apr

[23] M. Calvi, private communication, thesis in preparation 